\title{
CtBP2 proteome: Role of CtBP in E2F7-mediated repression and cell proliferation
}

\author{
Ling-Jun Zhao ${ }^{1}$, T. Subramanian ${ }^{1}$, S. Vijayalingam ${ }^{1}$, and G. Chinnadurai ${ }^{1}$ \\ ${ }^{1}$ Institute for Molecular Virology, Saint Louis University Health Sciences Center, Doisy Research Center, St. Louis, Missouri \\ Correspondence to: G. Chinnadurai, email: chinnag@slu.edu \\ Keywords: CtBP2; Proteome; E2F7; E2F1; NuRD \\ Received: August 23, $2013 \quad$ Accepted: April 21, 2014 Published: April 21, 2014
}

This is an open-access article distributed under the terms of the Creative Commons Attribution License, which permits unrestricted use, distribution, and reproduction in any medium, provided the original author and source are credited.

\section{ABSTRACT:}

C-terminal binding protein (CtBP) family transcriptional corepressors include CtBP1 and CtBP2. While CtBP1 and CtBP2 share significant amino acid sequence homology, CtBP2 possesses a unique $\mathrm{N}$-terminal domain that is modified by acetylation and contributes to exclusive nuclear localization. Although CtBP1 and CtBP2 are functionally redundant for certain activities during vertebrate development, they also perform unique functions. Previous studies have identified several CtBP1-interacting proteins that included other transcriptional corepressors, DNA-binding repressors and histone modifying enzymatic components such as the histone deacetylases and the histone demethylase LSD-1. Here, we carried out an unbiased proteomic analysis of CtBP2-associated proteins and discovered the association of several components of the CtBP1 proteome as well as novel interactions. The CtBP2 proteome contained components of the NuRD complex and the E2F family member E2F7. E2F7 interacted with the hydrophobic cleft region of CtBP1 and CtBP2 through a prototypical CtBP binding motif, PIDLS. E2F7 repressed E2F1 transcription, inhibited cell proliferation in a CtBP-dependent fashion. Our study identified CtBP as a corepressor of E2F7 and as a regulator of DNA damage response.

\section{INTRODUCTION}

CtBP 1 and CtBP2 are highly related transcriptional regulators and are implicated in a multitude of cellular functions. They (collectively referred here as CtBP) function predominantly as transcriptional co-repressors, in addition to certain diverse cytosolic functions (reviewed in $[1,2])$. In addition to transcriptional repression, invertebrate and vertebrate CtBPs also function as contextdependent transcriptional activators [3-5]. While CtBP1 is localized both in the cytosol and nucleus, CtBP2 is nuclear due to the presence of a unique 20 -amino acid N-terminal domain (NTR). The function of CtBP2 NTR is regulated by acetylation by p300 [6]. As inferred from their amino acid sequence homology and similarities in three dimensional structures $[7,8]$ (Pilka, ES et al., MMDB IP:4438), CtBP1 and CtBP2 are functionally redundant in the regulation of gene expression during animal development. However, they also perform unique developmental functions. Mice deficient in CtBP1 were viable, albeit, with reduced lifespan while CtBP2 null mice exhibited developmental defects beyond E10.5 and were not viable [9]. Differential interaction of CtBP cofactors may contribute to the functional difference between CtBP1 and CtBP2.

Between CtBP1 and CtBP2, the interacting proteins of $\mathrm{CtBP} 1$ have been more extensively characterized. A proteomic analysis of CtBP1-associated proteins revealed interaction with DNA binding repressors such as ZEB, corepressors such as CoREST and Znf217, class I histone decetylases 1 and 2 and the histone demethylase LSD-1 [10]. Mutational analysis of the interaction of different CtBP1-interacting proteins led to a model that the CtBP1 dimer may interact with promoter-bound repressors with one of the two hydrophobic clefts of the dimer while the other hydrophobic cleft region may interact with various histone modifying enzymes either directly or through other corepressors [11]. In addition to the proteomic analysis, other protein interaction studies have also identified other CtBP1-binding proteins under various contexts. In 
contrast to $\mathrm{CtBP} 1$, only limited attempts have been made to identify $\mathrm{CtBP} 2$-interacting proteins which resulted in the identification of proteins such as the tumor suppressor proteins HDM2/MDM2 [12] and ARF [13]. Here, we have carried out an unbiased proteomic study to identify CtBP2-associated proteins. Our analysis has identified several novel CtBP2-interacting proteins which include E2F7 and components of the nuclear remodeling histone deacetylase (NuRD) complex [14]. We demonstrate that both $\mathrm{CtBP} 1$ and $\mathrm{CtBP} 2$ interact with E2F7 and play critical roles during E2F7-mediated repression of E2F1 and cell proliferation. We also provide evidence that CtBP2 preferentially interacts with p66-beta subunit of the NuRD complex in a manner dependent on the NTR of CtBP2.

\section{RESULTS}

\section{CtBP2-interacting proteins.}

To examine whether CtBP2 interacts with unique cellular factors, we generated a HeLa cell line stably expressing Flag-HA-tagged CtBP2 (FH-CtBP2). FH$\mathrm{CtBP} 2$ cells were either untreated or treated with TSA, an inhibitor of histone deacetylases, before cell lysates were prepared for purification of CtBP2-bound proteins by Flag and HA double affinity purification. The FH-CtBP2 protein complex was subjected to LC-MS analysis. Comparison of TSA-treated and -untreated samples revealed subtle quantitative differences in certain $\mathrm{CtBP} 2$-bound proteins. However, both preparations of FH-CtBP2 bound to the same set of proteins. These proteins included previously identified proteins that bind to CtBP1 (reviewed in [2]), as well as novel proteins (Table 1). Among the novel proteins, E2F7 and components of the Nucleosome Remodeling Deacetylase (NuRD) complex, including CHD4 and p66-beta (reviewed in [15], have recently been shown to play critical roles in cell cycle regulation and chromatin histone modifications. Interestingly, a candidate PIDLS $\mathrm{CtBP}$-interaction motif is present close to the N-terminus of E2F7, and a PLDLS-like motif PVDMS is also located in the NuRD component p66-beta. Potential PLDLS-like motifs are also present in the Zinc finger protein TRPS1 and the WD repeat containing protein C2ORF44; however, the cellular functions of these proteins remain to be examined.

\section{Specific interaction of CtBP2 with E2F7 and NuRD components.}

To confirm the interaction between $\mathrm{CtBP} 2$ and the identified components of the $\mathrm{CtBP} 2$ protein complex, lysates from the FH-CtBP2 cell line and normal HeLa cells were used for co-immunoprecipitation with the Flag antibody. Western blots of the precipitated proteins were performed with antibodies as indicated (Fig.1A, lanes 1 and 2). Since E2F7 expression is subject to regulation by DNA damage response [16-18], we analyzed the same set of proteins in cells treated with etoposide (Fig.1A, lanes 3 and 4). As shown, in HeLa cells E2F7 bound to the Flag antibody to some background levels (lane 1); however, in cells expressing FH-CtBP2 the bound E2F7 signal was much stronger (lane 2). Etoposide treatment enhanced the level of E2F7 expression in both HeLa and FH-CtBP2 cell lines (compare lanes 3 and 4 with lanes 1 and 2). Correspondingly, an increased amount of E2F7 was associated with FH-CtBP2 (lane 4). By this analysis, CtBP2 was also shown to interact with the NuRD components, HDAC2, CHD4 and p66-beta. Factors such as ZNF217/CoREST/LSD-1 which were identified in the CtBP1 proteome [10] also readily interacted with CtBP2 (data not shown). However, the interaction of these factors with CtBP2 did not seem to be significantly influenced by etoposide treatment.

To substantiate the interaction between CtBP2 and E2F7, we examined a separate clone of HeLa/FHCtBP2 and a HeLa/FH-CtBP1 clone (Fig.1B) by a similar approach. The results showed that both CtBP2 and CtBP1 interacted with E2F7 specifically (Fig.1B, top panel) since the control HeLa cells were negative for the assay (lanes 1 and 4). To ascertain if endogenous CtBP2 interacts with E2F7, normal A549 cells and A549 cells with CtBP1 \& 2 double knock-down were used to prepare lysates, which were then immunoprecipitated with a CtBP2 antibody (Fig.1C, lanes 1 and 2). As shown, CtBP2 antibody pulled out E2F7 only from the normal A549 cells (lane 1) but not from the CtBP1 \& 2 knock-down cells (see Fig.4A for CtBP1\&2 Western blots), suggesting that endogenous $\mathrm{CtBP} 2$ and E2F7 interact with each other.

\section{E2F7 interacts with CtBP through a canonical CtBP binding motif.}

CtBP interaction with E2F7 was examined further by co-transfection of FH-CtBP expression constructs together with the Myc-tagged E2F7 expression construct (mouse E2F7 clone; [19] (Fig.1D). Cell lysates were co-immunoprecipitated with the Flag antibody and the precipitated proteins were examined by western blot analysis. In this analysis, both CtBP1 and CtBP2, as well as the CtBP2 mutant A58E [mutation located within the hydrophobic region that binds to PLDLS-like motifs [20]] were examined. As shown, both CtBP1 (lane 2) and CtBP2 (lane 3) bound to Myc-E2F7, and yet the A58E-CtBP2 mutant did not (lane 4), suggesting that CtBP interaction with E2F7 requires a PLDLS-like motif. Visual examination of the E2F7 sequence revealed the presence of a canonical CtBP-binding motif, PIDLS at the $\mathrm{N}$-terminal region. To examine the role of this motif in 
E2F7 interaction with CtBP2, we generated a Myc-E2F7 mutant with DL $\rightarrow$ AS mutation within the PIDLS motif. A similar mutation within the prototypical CtBP-binding protein, adenovirus E1A has been shown to abolish CtBP interaction [21, 22]. This mutant failed to depend on CtBP2 (Fig. 1D, lane 5). These results suggest that E2F7 interacts with CtBP2 through a canonical CtBP-binding motif with the hydrophobic cleft region of CtBP2.

\section{Unique interaction of p66-beta of the NuRD complex with CtBP2.}

In an attempt to determine whether CtBP1 and CtBP2 interact with any identified proteins differently, we transfected Flag-HA-tagged CtBP1 and CtBP2 expression constructs into HeLa cells and examined their interaction with different proteins. As shown in Fig. 2A, both CtBP1 and CtBP2 interacted with endogenous E2F7 and HDAC2. In contrast, the interaction with p66-beta was detected only with CtBP2 (lane 3). In addition, the A58E-CtBP2 mutant, which does not interact with PLDLS-like motifs, did not interact with any of these proteins. Thus, interaction with the NuRD component, p66-beta is unique to CtBP2 and appears to interact with the hydrophobic cleft region of CtBP2.

Since both CtBP1 and CtBP2 interacted with majority of partner proteins through PLDLS-like motifs and yet CtBP1 did not bind to p66-beta, we wondered whether the unique sequence in the CtBP2 N-terminal
A

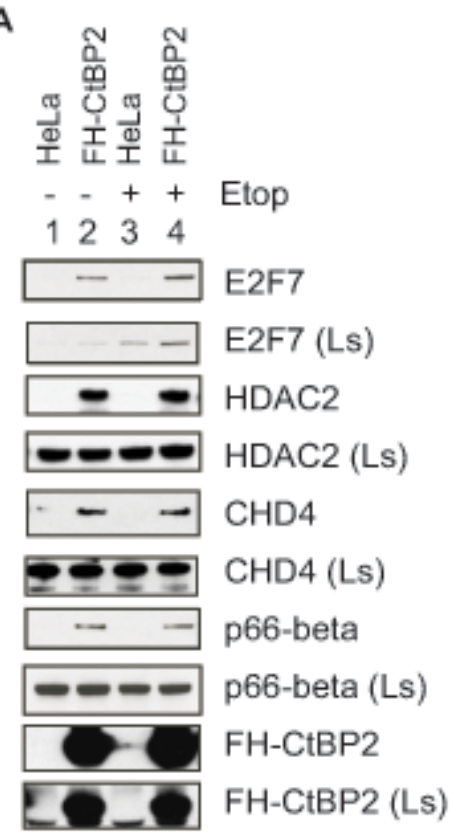

C

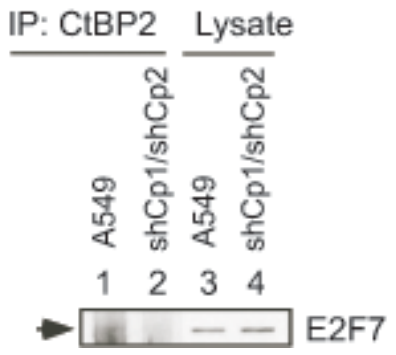

B

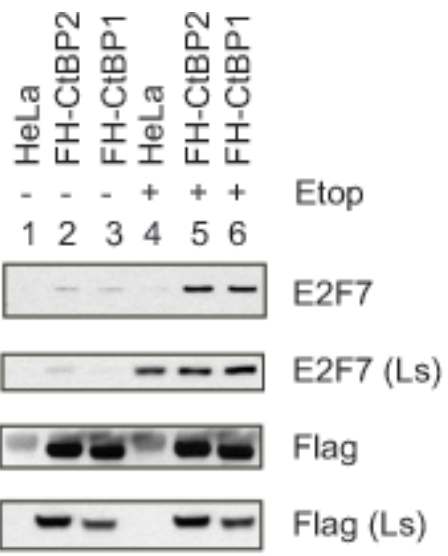

D

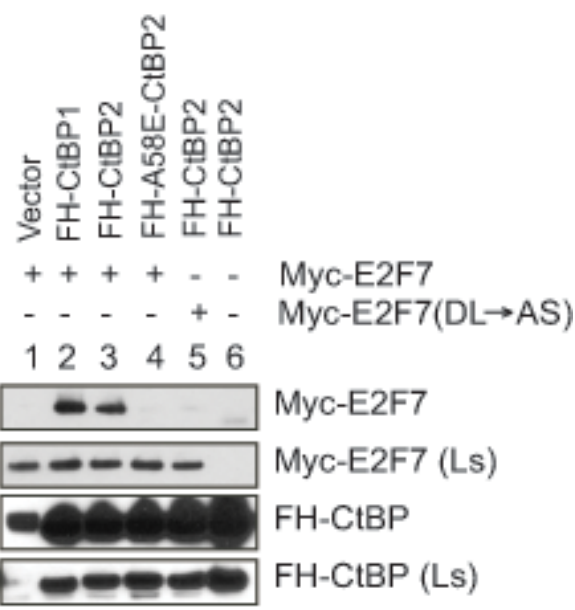

Figure 1: CtBP interaction with E2F7 and other proteins. A. HeLa and HeLa/FH-CtBP2 cell line (cell line \#1) which stably expresses Flag-HA-tagged CtBP2 were untreated or treated with $10 \mu \mathrm{M}$ etoposide for $6 \mathrm{hr}$, and then analyzed for binding of FH-CtBP2 to E2F7 and other cellular proteins by coimmunoprecipitation of cell lysates with Flag antibody beads followed by Western blot analyses with indicated antibodies. Ls: Cell lysate.B. FH-CtBP2 (cell line \#2), FH-CtBP1 and HeLa cells were treated with etoposide and immunoprecipitation and Western blot analyses were carried out as in A. C. A549 cells and CtBP1/2 down-regulated A549 cells were lysed and immunoprecipitated with CtBP2 antibody and analyzed by Western blotting using E2F7 antibody.D. FH-tagged CtBP constructs and Myc-E2F7 constructs were co-transfected into HeLa cells in combinations as indicated, and the interaction between CtBP and Myc-E2F7 proteins was examined as in A, except that $2 \mu \mathrm{g}$ of a non-specific IgG was used during co-immunoprecipitation with the Flag antibody beads to reduce non-specific binding of Myc-E2F7 to the Flag antibody beads. Ls: Cell lysate. 
Table 1: List of CtBP2-bound proteins. Numbers displayed for the cell lines are number of unique peptides identified.

\begin{tabular}{|c|c|c|c|c|c|}
\hline CtBP2 binding proteins (previously identified for CtBP1) & $\begin{array}{l}\text { Accession } \\
\text { Number }\end{array}$ & $\begin{array}{l}\text { Molecular } \\
\text { Weight }\end{array}$ & HeLa & $\mathrm{CtBP} 2$ & $\begin{array}{l}\text { CtBP2 } \\
(+\mathrm{TSA})\end{array}$ \\
\hline C-terminal-binding protein 2 isoform 1 & gi|4557499 & $49 \mathrm{kDa}$ & 2 & 42 & 48 \\
\hline Zinc finger protein 217 & gi|5730124 & $115 \mathrm{kDa}$ & 0 & 32 & 47 \\
\hline C-terminal-binding protein 1 isoform 1 & gi|4557497 & $48 \mathrm{kDa}$ & 2 & 19 & 21 \\
\hline Amine oxidase (flavin containing) domain 2, isoform CRA_a & gi|119615437 & $100 \mathrm{kDa}$ & 0 & 15 & 24 \\
\hline Zinc finger E-box-binding homeobox 1 isoform a & gi $\mid 189409130$ & $124 \mathrm{kDa}$ & 0 & 14 & 14 \\
\hline Adenomatous polyposis coli & gi| 182397 & $312 \mathrm{kDa}$ & 0 & 11 & 13 \\
\hline Histone deacetylase 1-like isoform 6 & gi|13138860 & $55 \mathrm{kDa}$ & 0 & 10 & 13 \\
\hline Histone deacetylase 2-like isoform 2 & gi|293336691 & $55 \mathrm{kDa}$ & 0 & 8 & 7 \\
\hline Ligand-dependent corepressor isoform 1 & gi|282847504 & $47 \mathrm{kDa}$ & 0 & 12 & 11 \\
\hline REST corepressor 1 & gi|344925845 & $53 \mathrm{kDa}$ & 0 & 3 & 11 \\
\hline Ligand dependent nuclear receptor corepressor-like isoform 1 & gi|260764001 & $67 \mathrm{kDa}$ & 0 & 3 & 5 \\
\hline \multicolumn{6}{|l|}{ Novel CtBP2 binding proteins } \\
\hline Transcription factor E2F7 & gi|145580626 & $100 \mathrm{kDa}$ & 0 & 4 & 4 \\
\hline Mi-2 protein (CHD4) & gi|1107696 & $218 \mathrm{kDa}$ & 0 & 13 & 16 \\
\hline Transcriptional repressor p66-beta & gi|21218438 & $65 \mathrm{kDa}$ & 0 & 11 & 10 \\
\hline Methyl-CpG binding domain protein 3, isoform CRA_b & gi $\mid 119589885$ & $26 \mathrm{kDa}$ & 0 & 3 & 5 \\
\hline Metastasis-associated gene & gi| 1008544 & $81 \mathrm{kDa}$ & 0 & 5 & 3 \\
\hline Metastasis-associated protein MTA2 & gi|14141170 & $75 \mathrm{kDa}$ & 0 & 11 & 14 \\
\hline Metastasis associated 1 family, member 3 , isoform CRA_d & gi|119620722 & $59 \mathrm{kDa}$ & 0 & 3 & 3 \\
\hline Retinoblastoma binding protein 7 , isoform CRA_b & gi|119619326 & $52 \mathrm{kDa}$ & 0 & 4 & 2 \\
\hline Zinc finger transcription factor TRPS1 & gi|6684534 & $142 \mathrm{kDa}$ & 0 & 8 & 10 \\
\hline WD repeat-containing protein C2orf44 isoform 1 & gi|13376798 & $79 \mathrm{kDa}$ & 0 & 6 & 7 \\
\hline Chromosome 20 open reading frame 112 , isoform CRA_b & gi|119596774 & $64 \mathrm{kDa}$ & 0 & 6 & 6 \\
\hline Human Dead-Box Rna Helicase Ddx3x & gi|114794734 & $47 \mathrm{kDa}$ & 0 & 2 & 2 \\
\hline Rho GTPase-activating protein 21 & gi|203097003 & $217 \mathrm{kDa}$ & 0 & 1 & 2 \\
\hline
\end{tabular}

region (NTR) contributes to this specificity. The CtBP2 mutant $\triangle \mathrm{N}-\mathrm{CtBP} 2$ which lacks the NTR, and N2-CtBP1 mutant in which the CtBP1 N-terminus is modified to contain CtBP2 NTR, were then transfected into HeLa cells and their interaction with co-factors was examined. As shown in Fig. 2B, deletion of the NTR from CtBP2 abolished CtBP2 interaction with p66-beta (lane 3) while maintaining $\mathrm{CtBP} 2$ interaction with HDAC2. Interestingly, acquisition of CtBP2 NTR rendered CtBP1 capable of interaction with p66-beta (lane 4). Thus, CtBP2 interaction with p66-beta appears to require both the NTR and the PLDLS-binding cleft of CtBP2.

We previously demonstrated that the unique CtBP2 NTR renders CtBP2 capable of nuclear localization as well as the ability to be acetylated [6]. One of the Lys residues within the NTR, Lys10, appeared to be critical for acetylation as well as CtBP2 nuclear localization. To examine if this residue is also critical for $\mathrm{CtBP} 2$ interaction with p66-beta, we transfected HeLa cells with the K10R-CtBP2 mutant and found that K10R mutation abolished CtBP2 interaction with p66-beta (Fig. 2B, lane 5). Thus, both CtBP2 nuclear localization and ability to interact with p66-beta require Lys 10 residue of CtBP2.

\section{Role of CtBP in E2F7-dependent repression of E2F1.}

E2F7 has recently been identified as a p53responsive E2F family member that is activated during DNA damage response $[17,18]$. E2F7 has also been identified as the repressor that regulates E2F1 expression 
[16-18]. Therefore, it is logical to expect that CtBP may serve as the corepressor of E2F7 to repress E2F1. To determine the role of CtBP in E2F7-dependent repression of E2F1, first we carried out a luciferase reporter assay. For this purpose, we generated a luciferase reporter construct which contains an approximately $300-b p$ promoter region of E2F1 (Fig. 3A), including the two E2F binding sites. HeLa cells were transfected with the E2F1-Luc reporter and $\mathrm{E} 2 \mathrm{~F} 7 \mathrm{wt}$ or $\mathrm{E} 2 \mathrm{~F} 7(\mathrm{DL} \rightarrow \mathrm{AS})$ mutant and the luciferase activity was determined (Fig. 3B). Transfection of E2F7 wt efficiently repressed the E2F1 promoter activity. In contrast, E2F1 promoter repression by E2F7 was significantly reduced by the DL $\rightarrow$ AS mutation.

We also determined the effects of endogenous CtBP1 and CtBP2 on the E2F1 promoter activity by using A549 cells that were acutely depleted of both CtBP1 and CtBP2 by infection with lentiviral vectors that express shRNAs against $\mathrm{CtBP} 1$ and $\mathrm{CtBP} 2$ followed by short term drug selection. As shown in Fig. 4A, CtBP1 and CtBP2 shRNAs caused efficient reduction in the expression of CtBP1 and CtBP2 levels (Lane 2). Similarly, we also generated shE2F7 (Fig. 4A, lane 3) and shE2F7/ shCtBP1\&2 triple knock-down cells (Fig. 4A, lane 4). The reduction in the levels of the intended target proteins was as expected as shown. Subsequently, E2F1wt-Luc or E2F1mt-Luc construct was transfected into normal A549 cells or the shRNA cell lines, and luciferase assays were performed to evaluate E2F1 promoter activity. As shown in Fig. 4B, knock-down of CtBP1\&2 enhanced E2F1 promoter activity, suggesting that $\mathrm{CtBP} 1 \& 2$ are important

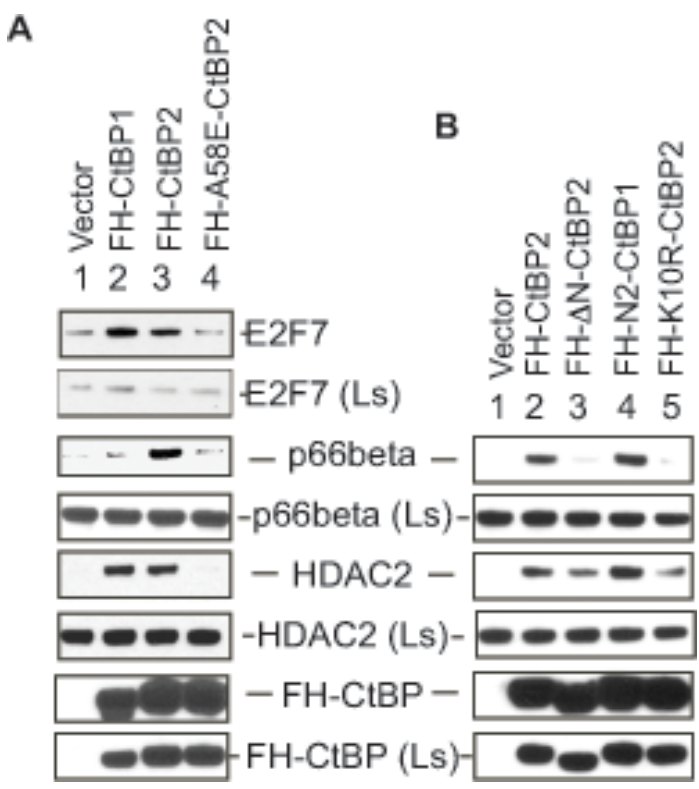

Figure 2: Unique interaction of CtBP2 with p66-beta. A. Role of CtBP2 hydrophobic cleft. B. Role of CtBP2 NTR. Flag-HA-tagged CtBP expression constructs were transfected into HeLa cells, and the interaction of the transiently expressed $\mathrm{CtBP}$ proteins with cellular proteins was analyzed as in Fig. 1. Ls: Cell lysate. for repression of E2F1 promoter. In contrast, knock-down of E2F7 alone did not have a significant impact. This result suggests that in the absence of E2F7, there may be alternative mechanisms to repress the $\mathrm{E} 2 \mathrm{~F} 1$ promoter. Alternatively, the cells might need to readjust to a reduced E2F7 level and recruit alternative repressors to regulate the E2F1 promoter. When E2F7 and CtBP1/2 were all knocked down, E2F1 promoter activity was also enhanced compared to normal A549 cells. However, the extent of the increase was not as high as when CtBP1\&2 were knocked down. Thus, in the absence of CtBP1 and 2, E2F7 might have lost its ability to repress E2F1 promoter. When the E2F1mt-Luc reporter DNA was used for transfection, the Luc activity was lower compared to the E2F1wt-Luc, suggesting that the observed effects of shRNAs on E2F1 promoter were most likely exerted through the two E2F elements in the E2F1 promoter, which are also the targets of E2F7.

The observation that CtBP1\&2 knock-down cells had a higher level of E2F1 promoter activity was consistent with CtBP1/2 being co-repressors for E2F7. To examine this possibility further, we co-transfected normal

A

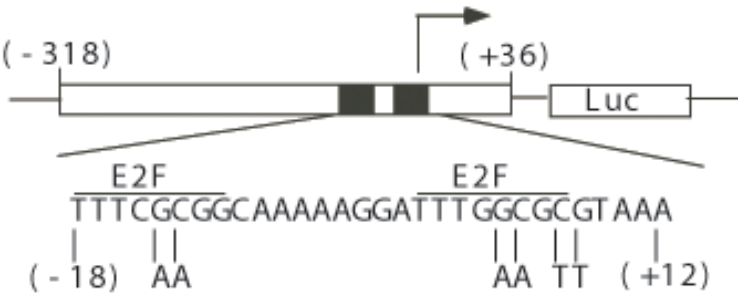

B

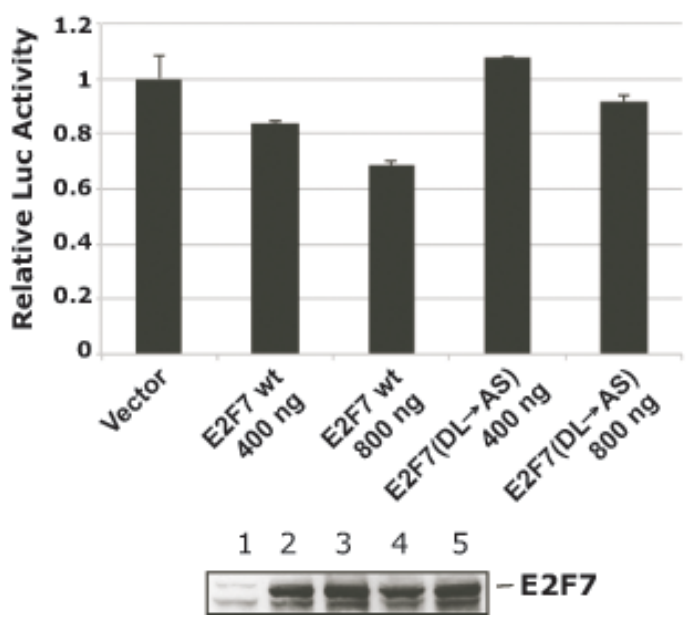

Figure 3: E2F1 promoter regulation by CtBP. A. Diagram of E2F1-Luc and E2F1-mu-Luc constructs. The E2F1 promoter region from (-)318 to (+)36 was PCR amplified and cloned into the pG5-Luc vector. The two E2F motifs in this promoter region were mutated as indicated at the bottom. B. E2F1-Luc was cotransfected with Myc-E2F7 or Myc-E2F7(DL $\rightarrow$ AS) mutant at indicated amounts, and Luc activity was determined. Only Luc data was plotted since Myc-E2F7 caused a severe repression of hRL-tk internal control. The expression of wt and mutant E2F7 proteins is shown at the bottom. 
A549 cells and the CtBP1/2 double knock-down cells with E2Fwt-Luc and E2F7 wt or E2F7(DL-AS). As expected, luciferase assays showed repression of E2F1-Luc in normal A549 cells by E2F7wt and much less efficiently by E2F7(DL-AS) (Fig. 5). In CtBP1\&2 double knockdown cells, over-expression of E2F7wt or E2F7(DL-AS) mutant still repressed the E2F1 promoter. This result could be partially due to the low level of CtBP1\&2 expression in the CtBP1/2 double knock-down cells. Alternatively, E2F7 could have additional mechanisms to repress E2F1 promoter in the absence of CtBP $1 / 2$.

\section{Effect of CtBP-E2F7 interaction on cell proliferation and cell cycle.}

To examine the functional significance of $\mathrm{CtBP}$ E2F7 interaction, we determined the effect of E2F7 wt and E2F7 (DL $\rightarrow$ AS) (defective in CtBP interaction) mutant on proliferation of U2OS cells (Fig. 6A). Transfection of E2F7 wt into U2OS cells resulted in substantial

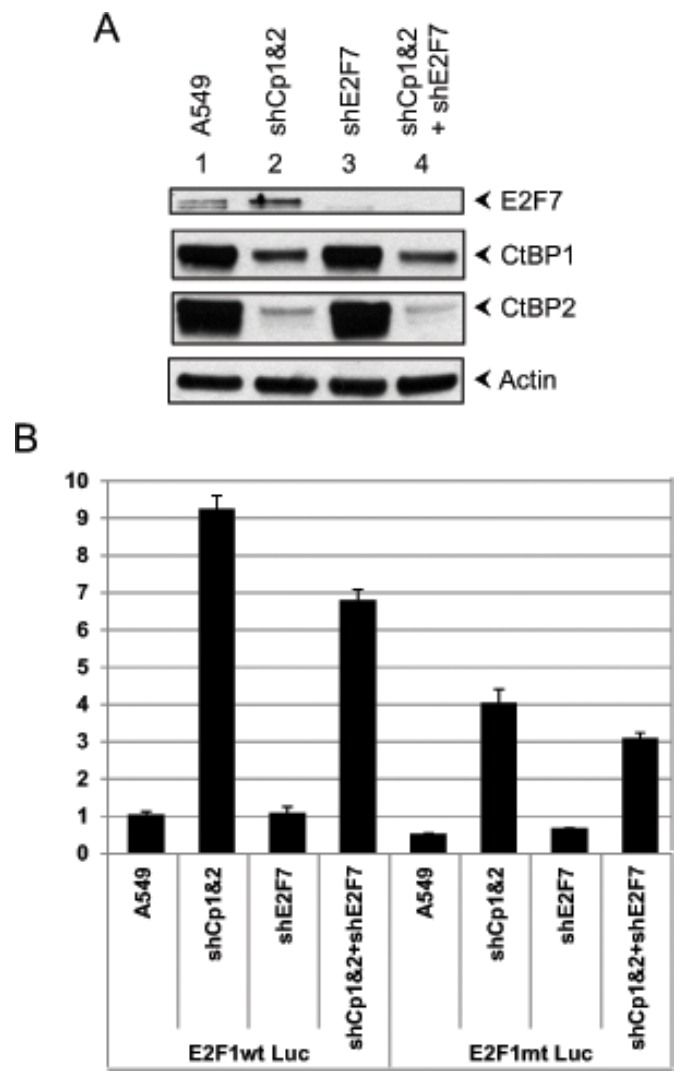

Figure 4: Effect of CtBP-depletion on E2F1 promoter activity. A. Protein expression profiles in A549 cells depleted for CtBP1/2 (shCp1\&2) and cells depleted for CtBP1/2 and E2F7 (shE2F7). Five days after lentiviral infection, cells were lysed and examined by Western blots. B. E2F1-wt-Luc and E2F1mu-Luc constructs were transfected into A549, shCtBP1\&2, shCtBP1/2+shE2F7 cell lines. Cells were lysed and luciferase assays performed. Experiment was performed in triplicates, and standard deviations are shown. reduction in colony formation while the E2F7(DL $\rightarrow$ AS) mutant generated more and bigger colonies (compared to cells transfected with E2F7 wt). These results suggest that the interaction of CtBP with E2F7 may contribute to the inhibition of cell proliferation.

Since E2F7 modulates DNA damage response through regulation of genes such as E2F1, U2OS cells with CtBP1 or CtBP2 knock-down were also used to determine the effect of $\mathrm{CtBP}$ on cell cycle regulation in response to treatment with etoposide (Fig. 6B). When subjected to cell cycle analysis, untreated CtBP1 knockdown cells appeared to have a G1 cell cycle block, while CtBP2 knock-down cells seemed to be similar to the control U2OS cells. After etoposide treatment, however, CtBP2 knock-down cells appeared to have an accelerated G1-S transition, as indicated by the higher proportion of cells at $\mathrm{S}$ phase and the lower proportion of cells at G1. In contrast, most of the CtBP1 knock-down-induced changes in untreated cells disappeared after etoposide treatment. Thus, knock-down of CtBP1 and CtBP2 appeared to affect the cell cycle differently, with CtBP2 knock-down exerting a more significant impact upon etoposide treatment.

\section{DISCUSSION}

By proteomic analysis, $\mathrm{CtBP} 2$ was found to interact
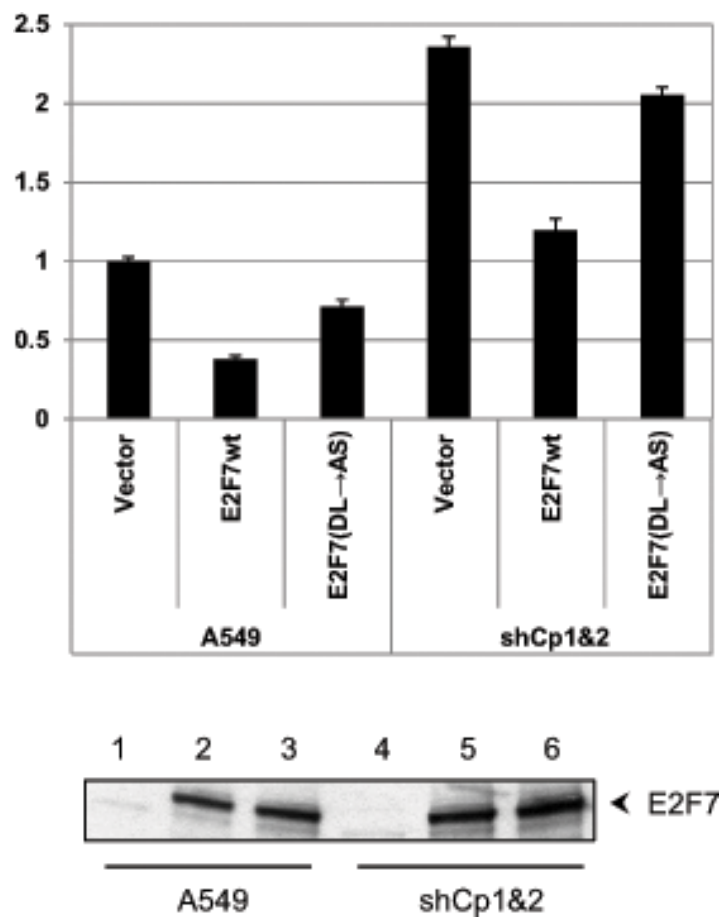

Figure 5: Effect of E2F7 overexpression on E2F1 promoter in CtBP-depleted cells. Cells were co-transfected with E2F1-Luc, hRL-tk and 800 ng of Myc-E2F7 or MycE2F7(DL $\rightarrow$ AS), and the luciferase assays performed. Bottom: Western blot for Myc-E2F7 with Myc antibody. 
with several new proteins in addition to certain previously identified proteins that bind to CtBP1 (reviewed in [2]) (Table 1). In the current study, we focused on E2F7 since it is the effector molecule of p53-mediated transcriptional repression and plays important roles in the expression of the critical trans-activating E2F family member, E2F1 that regulates cell proliferation and apoptosis [17, 18]. Although we identified E2F7 in the CtBP2-protein complex, we showed that it interacts with CtBP1 as well. During the preparation of this manuscript another report identified CtBP1 and CtBP2 as E2F7-interacting proteins [23]. Our results indicate that E2F7 interacts with the hydrophobic cleft region of $\mathrm{CtBP}$ since a mutation of a critical residue A58 of CtBP2 (corresponding to A52 of CtBP1) within the hydrophobic cleft region abolished E2F7 interaction. We also identified a prototypical CtBP binding motif PIDLS within the N-terminal region of E2F7 that mediates interaction with CtBP. Although presence of other CtBP-binding sequence elements within E2F7 cannot be ruled out, the $\mathrm{DL} \rightarrow \mathrm{AS}$ mutation within the core PIDLS motif abolished interaction with CtBP suggesting that this motif may be the primary binding sequence. We note that although the DL $\rightarrow$ AS mutation in E2F7 effectively abolished E2F7 interaction with CtBP (Fig. 1B), it only partially relieved the repressive activity of E2F7 in the transient reporter assay (Fig. 3) or E2F7A

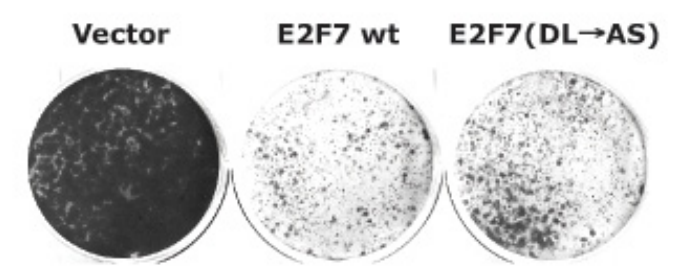

B

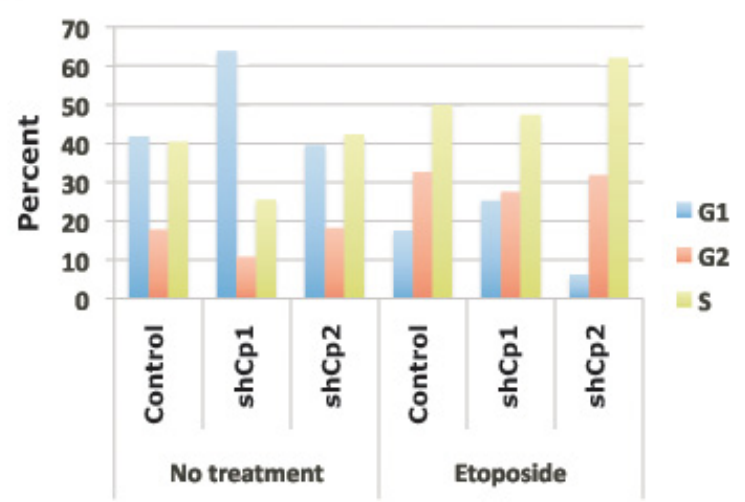

Figure 6: Effect of E2F7 and CtBP2 on cell proliferation and cell cycle regulation. A. Effect of E2F7 on cell proliferation. U2OS cells were transfected with vector, E2F7 wt or E2F7(DL $\rightarrow$ AS), and selected with $400 \mu \mathrm{g}$ / $\mathrm{ml}$ of G418 for 2 weeks. Drug-resistant cells were stained with crystal violet. B. Effect of CtBP2 depletion and etoposide on cell cycle. Cells depleted for CtBP1 (shCp1) or CtBP2 (shCp2) were either untreated or treated with etoposide $(10 \mu \mathrm{M})$ for $16 \mathrm{hr}$, and processed for cell cycle analysis. mediated inhibition of cell proliferation (Fig. 6A). These results suggest E2F7 may recruit other co-repressor(s), in addition to CtBP. They also raise the possibility that selective inactivation of $\mathrm{CtBP}$ functions may relieve the repressive action of E2F7 which could be exploited in chemotherapy regimens (see below).

Although E2F7 has been identified as a negative regulator of E2F1 and several other proliferation genes [18], the mechanism of transcriptional repression by E2F7 was not known. Our results suggest that CtBP may be a corepressor of E2F7. Thus, CtBP appears to be an important regulator of the E2F7-E2F1 regulatory loop that is activated by p53 during DNA damage and regulate cell proliferation and apoptosis. Since CtBP is overexpressed in several cancers $[17,24-30]$ targeting CtBP may enhance the chemotherapeutic effects of agents that activate p53 and E2F7. E2F7 overexpression has been identified as a prominent hall mark of squamous cell carcinomas (SCC) $[31,32]$. Since the relative chemoresistance of these carcinomas appears to be linked to high E2F7/E2F1 ratio, targeting CtBPs may enhance the expression of E2F1 and the apoptotic response. Since molecules that selectively disrupt CtBP functions are emerging [33, 34], such molecules may be exploited for the treatment of SCC.

Here, we have also identified different components of the NuRD complex in CtBP2 proteome. One of these components, p66-beta interacted with CtBP2 in a manner dependent on the N-terminal region (NTR) of CtBP2 suggesting that NuRD may be a CtBP2-specific repression effector. We have previously shown that acetylation of K10 within the NTR is essential for nuclear targeting of CtBP2 [6]. The interaction of p66-beta was also dependent of K10 (Fig. 2B). It is possible that CtBP2 NTR may play a more direct role in interaction with p66-beta other than nuclear targeting of CtBP2. Although a fraction of CtBP1 is normally localized in the nucleus, we did not detect significant interaction of p66-beta with nuclear localized CtBP1 by immunofluoresence analysis (not shown). Interestingly, in addition to the NTR, the interaction of p66-beta was also dependent on the hydrophobic cleft region since $\mathrm{CtBP} 2 \mathrm{~A} 58 \mathrm{E}$ mutant was deficient in interaction. We note that p66-beta contains a putative CtBP-binding motif PVDMS. Since the interaction of p66-beta with CtBP2 was generally stronger in coimmunoprecipitation experiments while the interaction of other NuRD subunits was variable, we believe that p66beta may the linker that connects NuRD with CtBP2. It is noteworthy that the activities of the NuRD complex are also linked to cancer, DNA replication and in DNA repair $[35,36]$. Future studies will determine the contexts in which $\mathrm{CtBP} 2$ may recruit the NuRD complex to regulate chromatin modifications and gene expression. 


\section{MATERIALS AND METHODS}

\section{Proteomic analysis.}

HeLa cells were transfected with pFH-CtBP2 plasmid [6] for $24 \mathrm{hr}$, trypsinized and plated at a low density in 6-well plates. After selection with G418 (400 $\mu \mathrm{g} / \mathrm{ml})$, well separated single cell colonies were cloned and expanded. Expression of FH-CtBP2 in the cell line was confirmed by Western blots as well as immunofluorescence analysis with Flag-Cy3 antibody. For proteomic analysis of CtBP2-interacting proteins, whole cell lysates from fifteen $100 \mathrm{~mm}$ dishes of $\mathrm{HeLa} /$ FH-CtBP2 cells were immunoprecipitated with the Flag antibody and the bound proteins were eluted with the Flag peptide, followed by a second step of purification with the HA antibody. Protein complexes bound to the HA antibody beads were directly subjected to trypsin digestion and proteomic analysis as described [37].

\section{DNA transfection for transient protein expression, lentivirus generation, and luciferase assays.}

For co-immunoprecipitation studies, HeLa cells were plated in $100 \mathrm{~mm}$ dishes at $1.6 \times 10^{6}$ cells per dish the day before transfection. HeLa, U2OS, and A549 cells were usually plated at $5 \times 10^{4}$ cells/well in 12-well plates for transfection to perform luciferase assays. All transfections were carried out with the XTreme HP transfection reagent (Roche) or Turbofect (Thermoscientific). To generate lentiviruses, 293T cells were plated in 6-well plates at $6 \times 10^{5}$ cells/well, and transfected the next day with a DNA mixture of $1.2 \mu \mathrm{g}$ shRNA plasmid (shCtBP1 or shCtBP2 plasmid,[38]), $1.2 \mu \mathrm{g}$ of pCMV- $\Delta 8.2 \Delta \mathrm{Vpr}$ (packaging DNA,[39]), and $0.6 \mu \mathrm{g}$ of pCMV-VSV-G. After transfection, cells were maintained for 2 days, and the culture supernatant $(2 \mathrm{ml})$ was filtered into $4 \mathrm{ml}$ DMEM supplemented with $10 \% \mathrm{FBS}$ and polybrene $(8 \mu \mathrm{g} / \mathrm{ml}$ final concentration), and used to infect cells in a $\mathrm{T} 75$ flask with $4 \times 10^{5}$ cells plated one day before infection. Selection of lentiviral infected cells (U2OS and A549 cells) was with $600 \mu \mathrm{g} / \mathrm{ml}$ of hygromycin B for shCtBP2, $6 \mu \mathrm{g} / \mathrm{ml}$ blasticidin for shCtBP1, or a combination of both drugs at $50 \%$ strength for dual selection of shCtBP1- and shCtBP2infected cells. E2F7 shRNA cells were selected with $1 \mu \mathrm{g} /$ $\mathrm{ml}$ of puromycin.

\section{Immunoprecipitation and western blot analysis.}

HeLa cells grown in $100 \mathrm{~mm}$ dishes were collected and lysed by freezing-thawing three times in $200 \mu$ l of a lysis buffer containing $20 \mathrm{mM}$ Tris- $\mathrm{HCl}$ (pH8.0), 0.3 $\mathrm{M} \mathrm{KCl}, 0.5 \mathrm{mM}$ EDTA, $10 \mathrm{mM} \mathrm{MgCl} 2,10 \%$ glycerol,
$0.1 \%$ NP40, 0.1\% Tween-20, $0.5 \mathrm{mM}$ DTT, $0.2 \mathrm{mM}$ PMSF, and Protease Inhibitor Complete Mini (Roche). After rotating at $4^{\circ} \mathrm{C}$ for $30 \mathrm{~min}$, cell debris was removed by centrifugation at $4^{\circ} \mathrm{C}$ for $5 \mathrm{~min}$. The clear cell lysate was diluted with $20 \mathrm{mM}$ Tris- $\mathrm{HCl}$ (pH8.0), and protein aggregates were removed again by centrifugation. The cell lysate was then mixed with $25 \mu \mathrm{l}$ of Flag-agarose beads (Sigma), and incubated at $4^{\circ} \mathrm{C}$ for $1 \mathrm{hr}$. In the case of FH-CtBP co-transfection with Myc-E2F7, before incubation with the Flag antibody beads, the cell lysate was first pre-incubated with $2 \mu \mathrm{g}$ of a mouse monoclonal antibody for luciferase (SCBT) to reduce non-specific binding of Myc-E2F7 to the Flag antibody beads. A 5 min centrifugation was done to remove potential protein aggregates. The antibody beads were washed twice with the cell lysis buffer diluted to $50 \%$ with $20 \mathrm{mM}$ Tris$\mathrm{HCl}(\mathrm{pH} 8.0)$. Bound proteins were eluted with a $2 \mathrm{X}$ SDS sample loading buffer without reducing agent for $10 \mathrm{~min}$ at room temperature. DTT $(100 \mathrm{mM})$ was added to the eluted proteins before loading to a $4-12 \%$ gradient gel (Life Technologies) for Western blot analysis.

\section{Cell cycle analysis.}

U2OS Cells were trypsinized, pelleted, washed with PBS, and fixed with $70 \%$ ethanol. After pelleting and washing with PBS, cells were stained for 30 min with a propidium iodide (PI) solution ( $20 \mu \mathrm{g} / \mathrm{ml}$ PI, $0.2 \mathrm{mg} / \mathrm{ml}$ RNaseA, $0.1 \%$ Triton X-100 in PBS), and subjected to cell cycle analysis on FACSCalibur.

\section{ACKNOWLEDGMENTS}

This work was supported by research grants CA84941 and CA-33616 from the National Cancer Institute. We thank Dr. Dana Madison for CtBP shRNA vectors and Dr. Gustavo Leone for Myc-E2F7 plasmid.

\section{REFERENCES}

1. Chinnadurai G. CtBP, an unconventional transcriptional corepressor in development and oncogenesis. Mol Cell. 2002;9:213-24.

2. Chinnadurai G. Transcriptional regulation by C-terminal binding proteins. Int J Biochem Cell Biol. 2007;39:1593607.

3. Fang M, Li J, Blauwkamp T, Bhambhani C, Campbell N, Cadigan KM. C-terminal-binding protein directly activates and represses Wnt transcriptional targets in Drosophila. EMBO J. 2006;25:2735-45.

4. Bhambhani C, Chang JL, Akey DL, Cadigan KM. The oligomeric state of CtBP determines its role as a transcriptional co-activator and co-repressor of Wingless targets. EMBO J. 2011;30:2031-43. 
5. Bajpe PK, Heynen GJ, Mittempergher L, Grernrum W, de Rink IA, Nijkamp W, et al. The Corepressor CTBP2 Is a Coactivator of Retinoic Acid Receptor/Retinoid X Receptor in Retinoic Acid Signaling. Mol Cell Biol. 2013;33:334353.

6. Zhao LJ, Subramanian T, Zhou Y, Chinnadurai G. Acetylation by p300 regulates nuclear localization and function of the transcriptional corepressor CtBP2. J Biol Chem. 2006;281:4183-9.

7. Nardini M, Spano S, Cericola C, Pesce A, Massaro A, Millo $\mathrm{E}$, et al. CtBP/BARS: a dual-function protein involved in transcription co-repression and Golgi membrane fission. EMBO J. 2003;22:3122-30.

8. Kumar V, Carlson JE, Ohgi KA, Edwards TA, Rose DW, Escalante CR, et al. Transcription corepressor CtBP is an NAD(+)-regulated dehydrogenase. Mol Cell. 2002;10:85769.

9. Hildebrand JD, Soriano P. Overlapping and unique roles for C-terminal binding protein 1 (CtBP1) and CtBP2 during mouse development. Mol Cell Biol. 2002;22:5296-307.

10. Shi Y, Sawada J, Sui G, Affar el B, Whetstine JR, Lan F, et al. Coordinated histone modifications mediated by a CtBP co-repressor complex. Nature. 2003;422:735-8.

11. Kuppuswamy M, Vijayalingam S, Zhao LJ, Zhou Y, Subramanian T, Ryerse J, et al. Role of the PLDLS-binding cleft region of $\mathrm{CtBP} 1$ in recruitment of core and auxiliary components of the corepressor complex. Mol Cell Biol. 2008;28:269-81.

12. Mirnezami AH, Campbell SJ, Darley M, Primrose JN, Johnson PW, Blaydes JP. Hdm2 recruits a hypoxiasensitive corepressor to negatively regulate $\mathrm{p} 53$-dependent transcription. Curr Biol. 2003;13:1234-9.

13. Paliwal S, Pande S, Kovi RC, Sharpless NE, Bardeesy N, Grossman SR. Targeting of C-terminal binding protein (CtBP) by ARF results in p53-independent apoptosis. Mol Cell Biol. 2006;26:2360-72.

14. Zhang Y, Ng HH, Erdjument-Bromage H, Tempst P, Bird A, Reinberg D. Analysis of the NuRD subunits reveals a histone deacetylase core complex and a connection with DNA methylation. Genes Dev. 1999;13:1924-35.

15. Allen HF, Wade PA, Kutateladze TG. The NuRD architecture. Cell Mol Life Sci. 2013.

16. Zalmas LP, Zhao X, Graham AL, Fisher R, Reilly C, Coutts $\mathrm{AS}$, et al. DNA-damage response control of E2F7 and E2F8. EMBO Rep. 2008;9:252-9.

17. Aksoy O, Chicas A, Zeng T, Zhao Z, McCurrach M, Wang $\mathrm{X}$, et al. The atypical E2F family member E2F7 couples the p53 and RB pathways during cellular senescence. Genes Dev. 2012;26:1546-57.

18. Carvajal LA, Hamard PJ, Tonnessen C, Manfredi JJ. E2F7, a novel target, is up-regulated by p53 and mediates DNA damage-dependent transcriptional repression. Genes Dev. 2012;26:1533-45.

19. de Bruin A, Maiti B, Jakoi L, Timmers C, Buerki R, Leone
G. Identification and characterization of E2F7, a novel mammalian E2F family member capable of blocking cellular proliferation. J Biol Chem. 2003;278:42041-9.

20. Zhao LJ, Subramanian T, Chinnadurai G. Changes in C-terminal binding protein 2 (CtBP2) corepressor complex induced by E1A and modulation of E1A transcriptional activity by CtBP2. J Biol Chem. 2006;281:36613-23.

21. Schaeper U, Boyd JM, Verma S, Uhlmann E, Subramanian T, Chinnadurai G. Molecular cloning and characterization of a cellular phosphoprotein that interacts with a conserved C-terminal domain of adenovirus E1A involved in negative modulation of oncogenic transformation. Proc Natl Acad Sci U S A. 1995;92:10467-71.

22. Subramanian $\mathrm{T}$, Zhao LJ, Chinnadurai G. Interaction of CtBP with adenovirus E1A suppresses immortalization of primary epithelial cells and enhances virus replication during productive infection. Virology. 2013;443:313-20.

23. Liu B, Shats I, Angus SP, Gatza ML, Nevins JR. Interaction of E2F7 with E2F1 and C-terminal Binding Protein (CtBP) Provides a Mechanism for E2F7-Dependent Transcription Repression. J Biol Chem. 2013.

24. Gujral TS, MacBeath G. A system-wide investigation of the dynamics of Wnt signaling reveals novel phases of transcriptional regulation. PLoS One. 2010;5:e10024.

25. Pena C, Garcia JM, Garcia V, Silva J, Dominguez $\mathrm{G}$, Rodriguez R, et al. The expression levels of the transcriptional regulators $\mathrm{p} 300$ and $\mathrm{CtBP}$ modulate the correlations between SNAIL, ZEB1, E-cadherin and vitamin D receptor in human colon carcinomas. Int $\mathrm{J}$ Cancer. 2006;119:2098-104.

26. Deng Y, Deng H, Liu J, Han G, Malkoski S, Liu B, et al. Transcriptional down-regulation of Brca1 and E-cadherin by CtBP1 in breast cancer. Mol Carcinog. 2012;51:500-7.

27. Di LJ, Fernandez AG, De Siervi A, Longo DL, Gardner K. Transcriptional regulation of BRCA1 expression by a metabolic switch. Nat Struct Mol Biol. 2010;17:1406-13.

28. Nadauld LD, Phelps R, Moore BC, Eisinger A, Sandoval IT, Chidester S, et al. Adenomatous polyposis coli control of C-terminal binding protein-1 stability regulates expression of intestinal retinol dehydrogenases. J Biol Chem. 2006;281:37828-35.

29. Deng H, Liu J, Deng Y, Han G, Shellman YG, Robinson $\mathrm{SE}$, et al. CtBP1 is expressed in melanoma and represses the transcription of p16INK4a and Brca1. J Invest Dermatol. 2013;133:1294-301.

30. Barroilhet L, Yang J, Hasselblatt K, Paranal RM, Ng SK, Rauh-Hain JA, et al. C-terminal binding protein-2 regulates response of epithelial ovarian cancer cells to histone deacetylase inhibitors. Oncogene. 2013;32:3896-903.

31. Endo-Munoz L, Dahler A, Teakle N, Rickwood D, HazarRethinam M, Abdul-Jabbar I, et al. E2F7 can regulate proliferation, differentiation, and apoptotic responses in human keratinocytes: implications for cutaneous squamous cell carcinoma formation. Cancer Res. 2009;69:1800-8. 
32. Hazar-Rethinam M, Cameron SR, Dahler AL, Endo-Munoz LB, Smith L, Rickwood D, et al. Loss of E2F7 expression is an early event in squamous differentiation and causes derepression of the key differentiation activator Sp1. J Invest Dermatol. 2011;131:1077-84.

33. Straza MW, Paliwal S, Kovi RC, Rajeshkumar B, Trenh P, Parker D, et al. Therapeutic targeting of C-terminal binding protein in human cancer. Cell Cycle. 2010;9:3740-50.

34. Birts CN, Nijjar S, Mardle C, Hoakwie F, Duriez PJ, Blaydes JP, et al. A cyclic peptide inhibitor of C-terminal binding protein dimerization links metabolism with mitotic fidelity in breast cancer cells. Chem Sci. 2013;4:3046-57.

35. Lai AY, Wade PA. Cancer biology and NuRD: a multifaceted chromatin remodelling complex. Nat Rev Cancer. 2011;11:588-96.

36. Li DQ, Kumar R. Mi-2/NuRD complex making inroads into DNA-damage response pathway. Cell Cycle. 2010;9:20719.

37. Komorek J, Kuppuswamy M, Subramanian T, Vijayalingam $\mathrm{S}$, Lomonosova E, Zhao LJ, et al. Adenovirus type 5 E1A and E6 proteins of low-risk cutaneous beta-human papillomaviruses suppress cell transformation through interaction with FOXK1/K2 transcription factors. J Virol. 2010;84:2719-31.

38. Madison DL, Lundblad JR. C-terminal binding protein and poly(ADP)ribose polymerase 1 contribute to repression of the p21(waf1/cip1) promoter. Oncogene. 2010;29:6027-39.

39. Lu Y, Liu C, Zeng L, Lin Z, Dewhurst S, Gartner S, et al. Efficient gene transfer into human monocyte-derived macrophages using defective lentiviral vectors. Cell Mol Biol (Noisy-le-grand). 2003;49:1151-6. 